\title{
Blogosphäre - soziale Netzwerke als Trendsetter
}

Das Web entwickelt sich zu einem neuen Lebensraum, in dem sich Nutzer präsentieren, mit anderen treffen, Informationen und Know-how austauschen, gemeinsame Projekte verfolgen und kulturelle Barrieren überwinden können. Der Beitrag gibt einen Überblick über Weblogs und Online-Gemeinschaften der Blogosphäre. Anhand verschiedener Praxisbeispiele wird aufgezeigt, wie in Bloggemeinschaften Trends gesetzt werden, die später wiederum von Suchmaschinen an die breite Öffentlichkeit getragen werden. Abrundend werden Handlungsempfehlungen für den Umgang mit sozialen Netzwerken der Blogosphäre präsentiert.

\section{Inhaltsübersicht}

1 Dimensionen sozialer Software

2 Weblogs

2.1 Soziodemografische Merkmale

2.2 Funktionsweise

3 Gemeinschaften der Blogosphäre

3.1 Online-Gemeinschaften und Sozialkapital

3.2 Auswirkungen von OnlineGemeinschaften auf Blogs

3.3 Multiplikationsfaktoren in Blogs und sozialen Netzwerken

4 Praxisbeispiele

4.1 Thematische Online-Gemeinschaften

4.2 Blogger als Medienpartner

5 Handlungsempfehlungen für die Praxis

6 Literatur

\section{Dimensionen sozialer Software}

Das heutige Web 2.o unterscheidet sich vom ursprünglichen Web nicht durch Technik, die in etwa gleich blieb, sondern durch soziale Aspekte, die jedermann befähigen, durch einfache
Bedienelemente selbst Daten ins Web zu stellen und sich mit anderen zu verknüpfen. Das Zauberwort dazu heißt soziale Software, also Software, die für die menschliche Kommunikation und Zusammenarbeit nützlich ist. Jeder sozialen Software ist gemein, dass sie dazu dient, Gemeinschaften aufzubauen und zu pflegen. Dabei kann soziale Software in drei Dimensionen aufgespaltet werden:

- Informationsmanagement: Ermöglicht das Finden, Bewerten und Verwalten von Information.

- Beziehungsmanagement: Ermöglicht Kontakte abzubilden, zu pflegen und neu zu knüpfen.

- Identitätsmanagement: Ermöglicht die Darstellung von Aspekten über sich selbst.

Weblogs, eine bestimmte Ausprägung von sozialer Software, können alle genannten Dimensionen abdecken. Gerade in neuster Zeit entstehen jedoch aus der Kombination von traditionellen Weblogs mit neuen sozialen Netzwerken (einer weiteren Ausprägung sozialer Software) immer effizientere Möglichkeiten für die breitere Bekanntmachung eines Blogartikels. Durch Ausnutzung sogenannter Multiplikationseffekte können dadurch vermehrt Trends aus der Blogosphäre an die breite Öffentlichkeit gelangen.

\section{Weblogs}

Eine einheitliche Definition, was ein Weblog, oder kurz Blog, ist, gibt es nicht. Meistens versteht man darunter aber eine spezielle Art eines regelmäßig aktualisierten Content-Management-Systems, das eine einfache Erstellung und Bearbeitung von Inhalten ermöglicht. Im Unterschied zu einem herkömmlichen Content- 
Management-System bestehen Weblogs normalerweise aus chronologisch rückwärts geordneten Einträgen von Kommentaren oder anderen Objekten wie Filmen, Bildern oder Diagrammen. Blogs können dabei die Form eines Tagebuchs, eines Journals, einer What's-New-Page oder einer Linksammlung annehmen. Generell sind alle Inhalte überwiegend durch Links mit anderen Webseiten verknüpft und können durch den Leser kommentiert, referenziert und bewertet werden. Die Gesamtheit der zusammengeschlossenen Blogs wird Blogosphäre genannt.

Wesentliche Charakteristika eines Weblogs sind eine regelmäßige Aktualisierung, eine Chronologie, verlinkte Inhalte und die Möglichkeit, Kommentare hinterlassen zu können. Diese sehr offene Definition kann folglich auch Microblogs wie Twitter (www.twitter.com), Fotoblogs wie Flickr (www.flickr.com) und Picasa (www.picasa.com), Business-Netzwerkplattformen wie LinkedIn (www.linkedin.com) und Xing (www.xing.com), herkömmliche soziale Netzwerkplattformen wie Facebook (www.facebook.com) und sogar geolokationsbasierte soziale Netzwerkplattformen wie Gowalla (www. gowalla.com) und Foursquare (www.foursquare.com) miteinschließen. Alle diese Dienste erlauben nämlich in irgendeiner Art regelmäßige Updates (sei es als Text, Bild, Videos oder Lokation), Verlinkung von Inhalten (beispielsweise durch "Like«- und "Star Rating"-Buttons) und Kommentarfunktionen.

\subsection{Soziodemografische Merkmale}

Dieser Abschnitt dreht sich um Eigenschaften von Blogger. Um aufzuzeigen, wer heutzutage warum bloggt, wird an dieser Stelle auf die Soziodemografie und die verschiedenen Nutzungsmotive eingegangen. Dabei wird der Begriff des Bloggens, wie einführend dargelegt, sehr weit gefasst. An dieser Stelle werden deswegen auch Micro- und Fotoblogs sowie soziale Netzwerkplattformen betrachtet.

2006 hat eine Studie der Universität Bamberg unter 4.000 deutschsprachigen Bloggern einen hohen Ausbildungsstandard ergeben. So besaßen rund $30 \%$ einen Hochschulabschluss und etwa $43 \%$ Abitur. Zudem waren die deutschsprachigen Blogger mehrheitlich jung (am häufigsten zwischen 20 und 29 Jahren) und männlich; bei den Blogbetreibern unter 20 Jahren dominierte jedoch mit $66 \%$ das weibliche Geschlecht. Über die Hälfte der Befragten nutzten das Internet seit fünf bis zehn Jahren und über $25 \%$ waren 11 bis 20 Stunden pro Woche online [Schmidt \& Wilbers 2006].

2010 zeigt eine neuere Technorati-Umfrage bei 7.200 Bloggern eine Veränderung in der Blogosphäre auf. Während die Nutzung bei den unter 25-Jährigen zurückgeht, finden immer mehr "Erwachsene" ihren Weg dorthin. So gibt es mittlerweile am meisten Blogger zwischen 25 und 45 Jahren. Bei den sozialen Netzwerken haben die Jugendlichen zwar zugelegt, die Erwachsenen aber ebenso. Heute nutzen etwa 4 von 5 Bloggern neben ihrem Weblog auch Twitter [Sobel 2010].

Thomas Pfeiffer führte im März 2009 eine Onlineumfrage zur Nutzung von Twitter durch. Dabei wurden aus 2.779 brauchbaren Datensätzen das deutsche Durchschnittsalter der Nutzer bei 32 Jahren, der Männeranteil bei $74 \%$ und der Abiturientenanteil bei $78 \%$ ausgemacht. Laut dieser Umfrage betreiben zwei von drei Nutzern einen eigenen Blog und schreiben vor allem über Technik, das Web oder Privates. $50 \%$ der Nutzer stammen dabei aus der Medien- oder Marketingbranche und jeder Vierte ist Führungskraft oder Unternehmer [Pfeiffer 2009].

Rechnet man auch Facebook zu den Blogs, ergibt sich laut Hutter folgendes Bild: Die Nutzungsbandbreite ist größer; in Österreich sind $79,25 \%$, in der Schweiz $82 \%$ und in Deutschland $82,8 \%$ der Nutzer zwischen 18 und 63 Jahre alt. In der Schweiz liegen die Männer anteilsmäßig (50,35\%) vor den Frauen (49,65\%), in Deutschland sind die Männer (49,9\%) in etwa gleich vertreten wie die Frauen $(50,1 \%)$ und in Österreich liegt der Frauenanteil (50,24 \%) höher als der Männeranteil (49,76 \%) [Hutter 2010]. 
Es gibt viele Gründe zu bloggen. Während nach der Umfrage von Technorati im Durchschnitt die primäre Motivation der Selbstausdruck und der Austausch von Fachwissen sind, geben $39 \%$ von Corporate-Blog-Betreibern an, dass sie bloggen, um ein Echo in der Medienwelt zu erhalten. So führen denn auch $57 \%$ der selbstständig erwerbstätigen Blogger an, dass sie Blogs nutzen, um neue Kunden zu werben. Dabei messen sie ihren Erfolg vor allem durch den Besuch von Webaufrufen, wohingegen Hobbyblogger ihren Erfolg mehr an persönlicher Zufriedenheit messen. Insgesamt teilen die Befragten jedoch das Gefühl, dass das Bloggen einen positiven Einfluss auf ihr Leben hat: $63 \%$ geben an, dass sie durch Bloggen besser in Bereiche ihrer persönlichen Interessengebiete involviert werden und dadurch sogar Freunde gewinnen konnten, die sie jedoch nie persönlich getroffen haben (62\%) [Sobel 2010].

Gewiss spornt es viele Blogger neben Redseligkeit und Eitelkeit an, zum Meinungsführer bei bestimmten Themen zu avancieren. Zudem werden häufig erst nach der Erstellung eines eigenen Blogs, ausgelöst durch den sozialen Austausch und die intellektuelle Tätigkeit des Schreibens, weitere positive Effekte festgestellt. Weil in Beiträgen jeder seinen individuellen Stil finden kann, ist der Umgang in Blogs durchaus lockerer als in anderen schriftlichen Dokumenten. Auffallend ist, dass vielfach Gründe genannt werden, die dem Individuum dienen, sich selbst zu verwirklichen, und ihm helfen, sich in ein soziales Netzwerk einzubinden.

\subsection{Funktionsweise}

Um zu verstehen, wie ein Blog funktioniert, wird anhand von Abbildung 1 in zehn Schritten ein möglicher Funktionsablauf vorgestellt [Portmann 2008]. Je nach System kann dieser Ablauf jedoch variieren.

Nachdem sich ein Blogger im System angemeldet hat, kann er in einem entsprechenden Formular einen neuen Eintrag kreieren (1). Üblicherweise erstellt dann das System automatisch eine neue, permanente Webseite (2) und integriert den letzten Eintrag in eine chronologisch rückwärts sortierte Liste aller Einträge. Des Weiteren erneuert das System ein RSSFeed, durch das einem Leser erlaubt wird, die brisantesten Headlines und Kurzbeschreibungen automatisch herunterzuladen. Zu diesem Zweck braucht der RSS-Reader (3) einen Link auf den RSS-Feed. Gleichzeitig informiert das System durch einen Blogping einen Ping-Server (4), wie beispielsweise blo.gs (http://blo.gs), über das Update. (5) Suchmaschinen bekommen von diesen Servern dann in periodischen

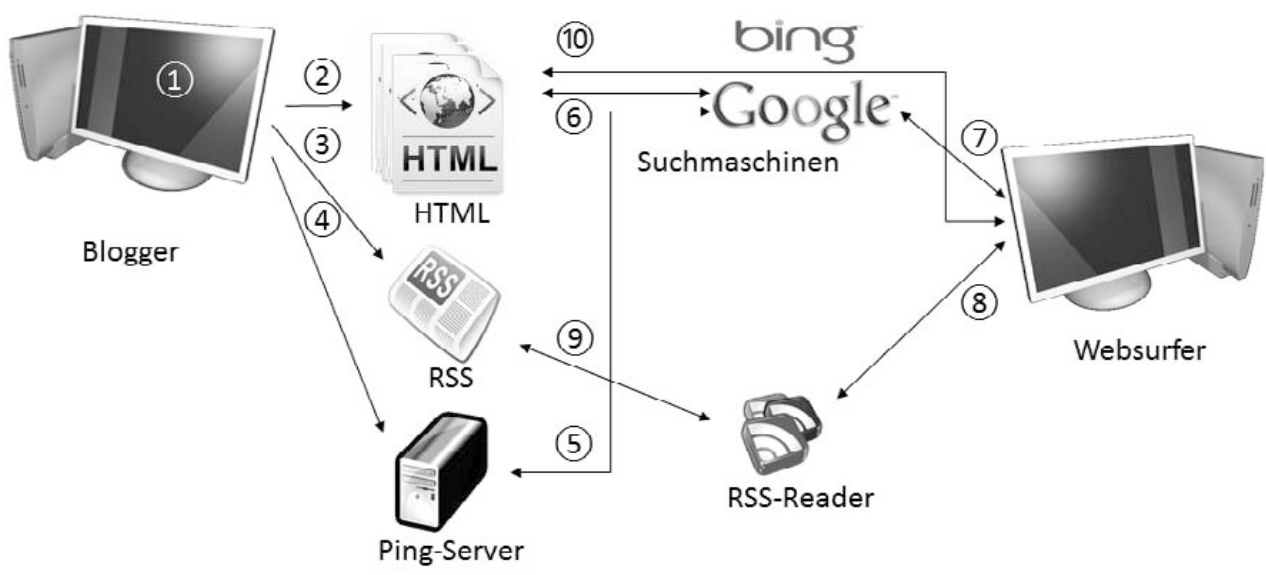

Abb. 1: Funktionsweise von Weblogs 
Abständen die entsprechenden Updates mitgeteilt, anhand derer diese (6) ihre Indizes neu ordnen und in ihre Datenbestände einarbeiten können.

In der Blogosphäre spricht man bezüglich Verlinkung oftmals von Track- oder Pingbacks, also von Funktionalitäten, mittels derer Informationen über Hyperlinks in Form von Reaktionen (Kommentare, Referenzen, Ratings usw.) durch einen automatischen Benachrichtigungsdienst zur Verfügung gestellt werden. In der Blogosphäre sind Track- und Pingbacks von zentraler Bedeutung, weil viele Blogs davon leben, auf andere Webseiten zu reagieren. Das machen sich auch Suchmaschinen zunutze und stufen oft verlinkte Seiten als wichtiger ein als weniger häufig verlinkte. Dabei sortieren die Suchmaschinen die Links dem Gewicht entsprechend, um dadurch eine Ergebnisreihenfolge bei einer Suchabfrage herzustellen, d.h., Links zu wichtigeren Seiten werden weiter vorne in der Ergebnisliste angezeigt. Durch eine Verlinkung von Blogs mit sozialen Netzwerken wird dieses Vorgehen der Gewichtung sogar noch stärker ausgenutzt.

Verschiedene Suchmaschinen erstellen neben den Suchergebnissen automatisch Webseiten, die eine Übersicht über neuste Beiträge in der Blogosphäre liefern. Der Websurfer erhält dadurch eine zusätzliche Möglichkeit, sich über aktuelle Weblogs zu informieren (7). Mittels des erwähnten RSS-Readers (8) können verschiedene Blogs durch ein einzelnes Programm auf neue Inhalte durchforstet werden. Dabei wird auf die von den Systemen automatisch erstellten RSS-Feeds (9) zurückgegriffen. Daraus ergibt sich folglich der Nutzen, dass nicht mehr von Hand jede Webseite aufgerufen werden muss (10), sondern dass diese über Lesezeichen erreicht werden können.

Bloggern werden durch eine fortwährend verbesserte Integration des sozialen SoftwareMixes immer weitere Möglichkeiten geboten, ihre Artikel zu bewerben. Augenfällig ist dabei, dass in neuster Zeit Weblogs immer mehr mit sozialen Netzwerken verknüpft werden. Im folgenden Abschnitt wird nun auf die durch verschiedene Funktionen geförderte Bildung von Gemeinschaften in der Blogosphäre eingegangen.

\section{Gemeinschaften der Blogosphäre}

Unter einer Gemeinschaft versteht man generell eine zu einer Einheit zusammengefasste Gruppe von Individuen, die emotionale Bindekräfte mit einem Zusammengehörigkeitsgefühl aufweist. Gemeinschaften können dabei von ihren Mitgliedern gegen Außenstehende abgegrenzt werden, ohne dass diese das notwendigerweise erkennen müssen.

\subsection{Online-Gemeinschaften und Sozialkapital}

Die Soziologen James Coleman und Pierre Bourdieu haben den Begriff Sozialkapital gebraucht, um die Einbettung von Individuen in soziale Strukturen zu umschreiben. Laut Pierre Bourdieu ist "das Sozialkapital [...] die Gesamtheit der aktuellen und potenziellen Ressourcen, die mit dem Besitz eines dauerhaften Netzes oder Anerkennens verbunden sind" [Bourdieu 1983]. Im Gegensatz zum Humankapital bezieht sich das Sozialkapital also nicht auf Personen, sondern auf die Beziehungen zwischen ihnen. Es bietet dabei einen Zugang zu Ressourcen wie Unterstützung, Anerkennung, Information, Wissen und Kontakte bis hin zum Finden von Arbeitsund Ausbildungsplätzen. Zum Beispiel kann eine Dynamik von Kennen und Anerkennen erklärt werden, wie sie etwa in Vereinen (Sport, Hobby etc.) und Serviceclubs (Rotary, Lions etc.) beobachtet werden können. Dabei kann aus dem Kennen einer Person ein Informationsvorsprung resultieren, wie beispielsweise das Wissen um eine neue, noch nicht offiziell ausgeschriebene Arbeitsstelle. In einem weiteren Schritt kann dann dieser Informationsvorsprung in einen Vertrauensvorschuss umfunktioniert werden, wenn sich der Bewerber nämlich 
gegenüber dem Personalchef auf diesen einen gemeinsamen Bekannten berufen kann.

Solche Ressourcennutzung ist auch in Online-Gemeinschaften zu beobachten. Als OnlineGemeinschaft wird dabei ein Kollektiv von Menschen bezeichnet, die einander im Web begegnen und sich über dieses austauschen. Ihre Kommunikation findet in einem sozialen Netz statt, das als Plattform zum gegenseitigen Austausch von Meinungen, Eindrücken und Erfahrungen dient. Diana Ingenhoff und Andreas Meier nennen dazu in [Ingenhoff \& Meier 2010] fünf Klassen verschiedener Webplattformen für die Online-Gemeinschaftsbildung:

- Gemeinschaftsnetzwerke: Sind elektronische Treffpunkte für Webnutzer, die einen gemeinsamen Ort, ein Interesse oder einen Lebensraum teilen.

- Buddy-Systeme: Zeigen auf, wo sich einzelne Freunde gerade befinden und wie sie elektronisch erreichbar sind.

- Matchmaking-Systeme: Erleichtern den Austausch von Beziehungen dadurch, dass Netzwerke von Freunden dazu verwendet werden, um Kontakte auf einer bereits vorhandenen Vertrauensbasis weiterzuknüpfen und Informationen austauschen zu können.

- Empfehlungssysteme: Mittels dieser Systeme werden die Vorlieben der Webnutzer eruiert und diesen werden Vorschläge zur Weiterentwicklung gemacht.

- Corporate-Blog-Systeme: Diese Systeme erlauben es, Wissen zu vermitteln, Themen zu besetzen oder Beziehungen zu pflegen.

Alle diese Plattformen können einzelnen Dimensionen von sozialer Software zugeordnet werden. Zudem haben diese Plattformen gemeinsam, dass es sich um Austausch-, Diskussions- und Kommunikationsplattformen einer Online-Gemeinschaft handelt. Dabei gilt es, zu beachten, dass jedwede Online-Gemeinschaft aufgebaut, gepflegt und betreut werden muss, wozu oft Mitglieder für verschiedene Aufgaben mit einbezogen werden. Im Journalismus beispielsweise spielen Leser-, Hörer- und Zuschauer-Gemeinschaften bei der Leser-BlattBindung eine wichtige Rolle. Zu Beginn wurden Online-Gemeinschaften oft mit der von ihr verwendeten Technik gleichgestellt. Heute ist man jedoch der Ansicht, dass sich die Gemeinschaften nicht über Technik definieren, sondern über zusammenführende Themen, also ein soziales Phänomen darstellen.

Durch Blogs und deren Verlinkung untereinander können Blogger Online-Gemeinschaften mit gleichartigen Themen, beispielsweise Gadgets, Digitalfotografie, Fashion, Gastronomie, Sport, Musik usw., bilden. Dabei wird die Online-Gemeinschaftsbildung durch die für Leser geschaffene Funktion, Kommentare mit Links zu eigenen Blogs zu hinterlassen, gefördert. Weil heutzutage Blogs zudem häufig mit sozialen Netzwerken verknüpft werden, wirkt hier ein Multiplikationseffekt. Deshalb können in der Gemeinschaft der Blogosphäre Trends gesetzt werden, die zum einen durch die starke Verlinkung in dieser selbst und zum anderen durch eine geeignete Verlinkung mit OnlineGemeinschaftsplattformen von Suchmaschinen an die breite Öffentlichkeit gelangen und diese beeinflussen.

\subsection{Auswirkungen von Online- Gemeinschaften auf Blogs}

Am Beispiel der marktführenden OnlineGemeinschaftsplattform Facebook lässt sich sehr gut illustrieren, wie erfolgreiche Web-2.0Konzepte nicht mehr nur an eine Plattform geknüpft sind, sondern als Quasistandards von zeitgenössischen Onlineplattformen eingesetzt werden. Das generische Basiskonzept von Facebook wird bereits von zahlreichen anderen Plattformen wie Online-Business-Netzwerken verwendet. Im Rahmen einer geschlossenen Benutzergruppe kann ein persönliches Profil mit Daten über beispielsweise Lebenslauf, Bildungsweg, persönliche und geschäftliche Interessen sowie Kontaktmöglichkeiten erfasst werden. Das persönliche Profil wird vernetzt mit 
anderen Benutzerprofilen von Bekannten und Geschäftspartnern, mit denen man somit über Kurznachrichten oder E-Mail in Kommunikation treten kann. Das persönliche Profil ist mehrheitlich nicht öffentlich zugänglich, sondern steht ausschließlich den Benutzern der Plattform offen, denen man dabei auch noch explizit Zugang zu den persönlichen Daten gewähren muss.

Die neue Generation von Web-2.o-Plattformen ergänzt die Basisfunktionalitäten des Generischen um am Markt bereits erprobte Konzepte aus Blogs, Web-2.o-Plattformen und spezialisierten sozialen Mediendiensten, die im Nachgang skizziert werden.

Neben der Profilierung und Vernetzung verfügt Facebook über die typischen Kernfunktionalitäten eines herkömmlichen Blogs. Benutzer können Inhalte verschiedener Form (Text, Bilder, Video, Links) auf einer persönlichen Seite erfassen, die in chronologischer Abfolge dargestellt werden. Diese Einträge können kommentiert, referenziert und (durch "Like«-Buttons) bewertet werden.

2006 wurde Twitter, der bekannteste und derzeit am weitesten verbreitete MicrobloggingDienst, gegründet. Auf der Website können Kurznachrichten, sogenannte "Statusupdates", von bis zu 140 Zeichen im Stil von SMS auf dem eigenen Twitter-Profil veröffentlicht werden. Die Benutzung von Twitter erfolgt dabei mehrheitlich über mobile Endgeräte und weniger über die Eingabe via Webbrowser. Facebook übernahm 2010 dieses Konzept in Form eines zentralen Eingabefelds für die Erfassung von Inhalten auf der persönlichen Startseite und bietet seitdem darüber hinaus auch die Möglichkeit, Inhalte über mobile Applikationen standortunabhängig zu erstellen.

Gowalla (gegründet 2007) und Foursquare (gegründet 2009) sind die beiden führenden Unternehmen im Bereich der geolokationsbasierten sozialen Netzwerke. Die Benutzer deren Dienste können über mobile Endgeräte, die über globale Positionierungssystem-Funktiona- lität (GPS) verfügen, Orte in ihrer Umgebung mit Koordinaten speichern, mit einer Kurzmitteilung versehen und damit den aktuellen Aufenthaltsort ihrem Online-Netzwerk bekannt geben. 2010 integrierte Facebook dieses Konzept als "Places-Funktion « auch in seine eigene Plattform, ließ dabei aber gleichzeitig noch die Möglichkeit offen, dass Benutzer der Pionierplattformen Aktualisierungen ebenfalls nach Facebook publizieren können.

Die Integration von Web-2.o-Konzepten als Quasistandard auf sozialen Netzwerkplattformen führt dazu, dass den Benutzern einfache Instrumente mit sehr niedriger Kontributionsschwelle zur Veröffentlichung von Inhalten zur Verfügung stehen. Im Unterschied zur Bereitstellung und Konfiguration eines Weblogs braucht es für die Publikation auf diesen Plattformen keine speziellen technischen Kenntnisse. Waren es in den vergangenen Jahren hauptsächlich Blogger, die benutzergenerierte Inhalte geschrieben und den Bürgerjournalismus geprägt haben, sind es jetzt auch Gelegenheitsbenutzer, die selbst erstellte Inhalte veröffentlichen.

Die Verfügbarkeit der neuen Publikationsmittel und -kanäle wirkt sich mittlerweile auch auf das Medienverhalten der etablierten Blogger aus: Es ist zu beobachten, dass die Anzahl aktiver Blogs und die Publikationsfrequenzen tendenziell abnehmen. Blogger bedienen sich der neuen Instrumente, um die Inhalte präziser zu platzieren oder aufgrund einfacherer Benutzung, beispielsweise über mobile Endgeräte, standortunabhängig zu veröffentlichen. So werden heute Neuigkeiten oder Links auf tagesaktuelle Onlineinhalte aufgrund des verkürzten Publikationswegs eher in Echtzeit via Twitter oder Facebook veröffentlicht, wofür in der Vergangenheit ein ganzer Blogbeitrag geschrieben worden wäre. Herausgeber von Weblogs nutzen die neuen Dienste zudem aktiv, um ihre eigenen Blogbeiträge zu bewerben und weitere Leserschaft zu gewinnen. 


\subsection{Multiplikationsfaktoren in Blogs und sozialen Netzwerken}

Viele Online-Netzwerke verfügen über Funktionen, um die erstellten Inhalte einer breiteren Masse zu kommunizieren. Dies erfolgt einerseits innerhalb des eigenen Netzwerks, d.h. für die eigenen Kontakte in der Teilöffentlichkeit der geschlossenen Benutzergruppe, andererseits aber auch durch die Veröffentlichung auf Drittplattformen und Suchmaschinen nach dem Vorbild der Trackback/Ping-Funktionen der Blogs. Facebook verfügt dabei über einen "Like«-Button, mit dem Inhalte von anderen Autoren bewertet bzw. als lesenswert gekennzeichnet werden können. Dies ist eine einfache Form eines Empfehlungssystems. Die markierten Inhalte erscheinen auf den persönlichen Websites der verbundenen Benutzer in Form einer persönlichen Empfehlung. Die äquivalente Funktion bei Twitter ist das sogenannte "Retweeten", das Weiterleiten eines fremden Eintrags an die eigene Leserschaft.

Nach der Umfrage von Technorati verlinken $56 \%$ der Blogger, die auch Twitter benutzen, ihren Blog mit dem Twitter-Konto. $34 \%$ geben an, dass sich Twitter binnen Jahresfrist als effektiver Multiplikationskanal erwiesen hat. $87 \%$ der befragten Blogger verfügten über einen Facebook-Account. Die Befragten nennen Facebook und Twitter als mit Abstand die wichtigsten Plattformen für die Bekanntmachung ihrer Blogbeiträge [Sobel 2010].

Neben der Verwendung dieser beiden Dienste bieten sich bloggenden Autoren weitere Web-2.o-Dienste für die Veröffentlichung von spezifischen Dateiformaten. In der Praxis hat es sich eingebürgert, Multimediaformate nicht physisch auf der eigenen Bloginfrastruktur zu speichern, sondern bei spezialisierten Diensten wie z.B. YouTube (www.youtube.com) für Filme, SlideShare (www.slideshare.net) für PowerPoint-Dateien oder Flickr für Fotos hochzuladen. Damit sind verschiedene Vorteile verbunden. Multimediadateien müssen nicht in webgerechte Formate konvertiert werden, was mit erheblichem Zeitaufwand und erforderlichen Fachkenntnissen verbunden wäre. Zudem liegen die Dateien nicht auf dem eigenen Server und belegen somit keinen Speicherplatz und benötigen keine Netzbandbreite beim Aufrufen. Der größte Vorteil im Sinne der Reichweite von Leserschaft liegt aber in der Suchmaschinenrelevanz und der Möglichkeit, die Bilder, Videos oder Präsentationen auf Drittplattformen einzubinden. So wird YouTube mittlerweile als zweitgrößte Suchmaschine bezeichnet. Eine Google-Suche nach einem Stichwort bringt oft unter den ersten Treffern explizit passende Inhalte in Videoformat. Das bringt zusätzliche Leserschaft über Suchmaschinentreffer. Über kleine Programmcodeschnipsel, sogenannte Embed-Codes, können Blogger oder deren Leser die Multimedia-Inhalte auf ihren eigenen Blogs, Webseiten oder sozialen Mediennetzwerken einfügen, was zu einer viralen Verbreitung des Ursprungsinhalts führen kann.

Für Blogger entstehen aus der Kombination von traditionellen Weblogs mit sozialen Netzwerken und Web-2.o-Diensten effiziente Möglichkeiten für die breitere Bekanntmachung durch Multiplikationseffekte und die attraktivere Ausgestaltung ihrer Blogartikel mit multimedialen Inhalten.

Im folgenden Abschnitt werden nun verschiedene Beispiele aus der Praxis kurz erläutert.

\section{Praxisbeispiele}

Die Blogosphäre kann sich der rasanten Entwicklung der sozialen Netzwerke und der Web2.o-Plattformen nicht entziehen. Die große Popularität der marktführenden Plattformen, die niedrigen Kontributionsschwellen und Verbreitung von mobilen Endgeräten und Datennetzen führt zu einer zunehmenden Substituierung und Verwässerung der ursprünglichen Form der Weblogs. Erfolgreiche, am Markt bewährte Web-2.o-Konzepte und Blogfunktionalitäten 
sind mittlerweile integrale Bestandteile der sozialen Netzwerkplattformen geworden. Daneben etablieren sich Sonderformen von Blogs und Web-2.o-Diensten mit sich an Blogs anlehnenden Grundfunktionalitäten.

\subsection{Thematische Online-Gemeinschaften}

Die Vielzahl der Mitmachplattformen und deren Web-2.o-Funktionalitäten ermöglichen eine einfache Organisation von Gleichgesinnten in Online-Gemeinschaften. Die Gattung eines Dienstes hinsichtlich Dateiformat kann dabei eine thematische Nähe implizieren.

Flickr oder Picasa eignen sich als Fotoplattformen primär für die Organisation von Bildund Videomaterial. Diese Plattformen können dabei entweder vom Benutzer selbst für die Organisation der eigenen Werke oder auch, dank spezieller Eigenschaften, für den thematischen Zusammenschluss mit anderen Teilnehmern im selben Netzwerk verwendet werden. Daraus können sich selbst verwaltende Interessengemeinschaften mit Themen wie beispielsweise Schwarz-Weiß-, Analog-, Portrait- oder Architekturfotografie entstehen. Auch hier kommen die besprochenen Konzepte zur Anwendung: Kommentare, Bewertung, stabile Referenzierung sowie einfache Einbettungsmöglichkeit der Fotos auf Drittplattformen.

Von ähnlicher Form sind Dienste wie YouTube für die Veröffentlichung von Videomaterial, Deviantart (www.deviantart.com) im Bereich Kunst, Design und Literatur sowie SlideShare für PowerPoint-Präsentationen und Videos von Referaten.

Plattformen wie Facebook, Xing oder Linkedln hingegen beschränken sich nicht primär auf bestimmte Dateiformate. Sie haben darüber hinaus sogar zusätzliche Möglichkeiten, die sich für die Verwaltung und Administration einer Gemeinschaft eignen. Im Speziellen sind dies die Erstellung von Gruppen, die entweder öffentlich, teilöffentlich oder geschlossen nur auf Einladung sichtbar sind, und die Erstellung und Verwaltung von Veranstaltungen, die ebenso öffentlich oder teilöffentlich ausgeschrieben werden können.

Spannend zu beobachten ist, wie diese sowohl von verschiedenen Anspruchsgruppen aus Wirtschaft, Kultur, Kirche, Politik, Medien und gemeinnützigen Organisationen als auch von Privaten genutzt werden, um ihre Kommunikationsaktivitäten auf die neuen Kanäle auszuweiten.

Im aktuellen Wahljahr scheinen viele Parteien, Politiker und Kandidaten sich ein Beispiel an der US-Präsidentschaftskampagne zu nehmen und setzen Blogs, soziale Netzwerke und Web-2.o-Plattformen in unterschiedlicher Kombination ein. Die FDP Schweiz zum Beispiel lanciert Wahlkampfvideos auf YouTube und die CVP Schweiz bedient sich Facebook, Twitter und Flickr mit Text und Bild.

Abt Martin Werlen vom Kloster Einsiedeln unterhält aktiv einen Twitter-Account, um mit der Kirchenbasis auf eine moderne Weise zu kommunizieren, aber noch mehr, um mit Menschen in Kontakt zu kommen, die er über die üblichen Kanäle nicht erreichen könnte. Abbildung 2 zeigt einen Auszug aus Abt Martin Werlens Twitter-Account.

Ein ähnliches Bild zeichnet sich in der Schweizer Tagespresse ab. Die Neue Zürcher Zeitung und der Tagesanzeiger verwenden in ihren Onlineausgaben Blogs in ursprünglicher Form und darüber hinaus Multiplikationsfunktionen in Form von sozialer Medienintegration, hauptsächlich für Facebook und Twitter (vgl. Abb. 3). Die Blogs sind thematisch klar positioniert. Der Tagesanzeiger führt monothematische Blogs zum Thema Wirtschaft, Politik, Elternschaft, Lifestyle und Sport, während die Neue Zürcher Zeitung auf Blogs einzelner Autoren setzt - unter innen beispielsweise Schwester Rut-Maria Buschor aus dem Benediktinerinnenkloster St. Andreas in Sarnen -, die abseits der Tagesaktualität ganz persönliche Meinungen publizieren. Dies ist ganz im ursprünglichen Sinne der Blogs. Ebenfalls im ursprünglichen Sinne belassen die Medienhäuser die 


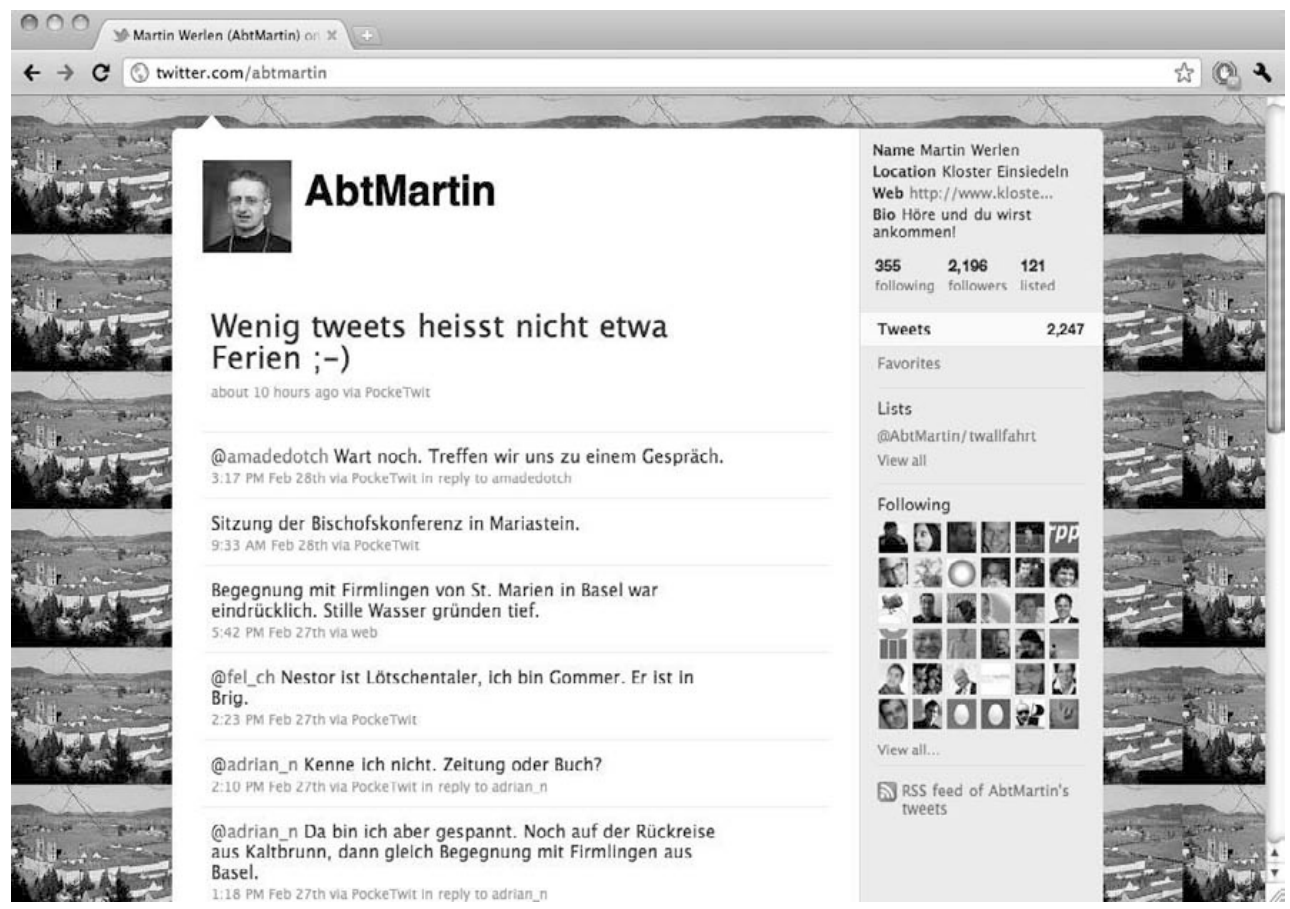

Abb. 2: Twitter-Account von Abt Martin Werlen vom Kloster Einsiedeln

Kommentarfunktionalitäten und Aggregationselemente wie RSS-Feeds.

Erwähnenswert sind auch Beispiele von gemeinnützigen Organisationen wie Amnesty International (www.amnesty.org) und Greenpeace (www.greenpeace.org). Amnesty International hat im Jahr 2010 binnen 14 Tagen über 10.000 virtuelle Kerzen und Bestellungen für 50.000 physische Postkarten generiert, die per Briefpost an das libysche Regime gesandt wurden, um die Freilassung von zwei inhaftierten Schweizer Geiseln zu fordern [Allemann 2010]. Greenpeace bewegte in einer noch nie dagewesenen internationalen Kampagne in Form einer Parodie auf ein bekanntes Nestlé Markenprodukt das Unternehmen dazu, künftig auf Palmöl aus dem Urwald zu verzichten, da dieses den Lebensraum von Orang-Utans zerstört [Borgerding 2010].

Beide gemeinnützigen Organisationen haben dank Nutzung von Standardfunktionalitä- ten von Blog und sozialen Medienplattformen eine temporäre Gemeinschaft für die Dauer der Kampagne bilden und mittels Multiplikationseffekten innerhalb kürzester Zeit große Reichweiten und mediale Aufmerksamkeit erzeugen können.

Nach demselben Muster sind private Initiativen im Vorfeld von politischen Abstimmungen zu beobachten. Facebook-Gruppen und TwitterAccounts werden mit dem Ziel erstellt, auf politische Themen aufmerksam zu machen, Positionen und Statements über die privaten Kanäle der Benutzer zu verbreiten und Wählerschaft zu mobilisieren. In jüngster Vergangenheit wurden Blogs und soziale Medien gar als hauptsächliche Medien für die Organisation von Protestbewegungen im arabischen Raum verwendet. Diese Kanäle entpuppten sich für westliche Journalisten als wertvolle parallele Informationsquellen in Echtzeit als Alternative zu den staatlichen, der Propaganda unterworfenen 


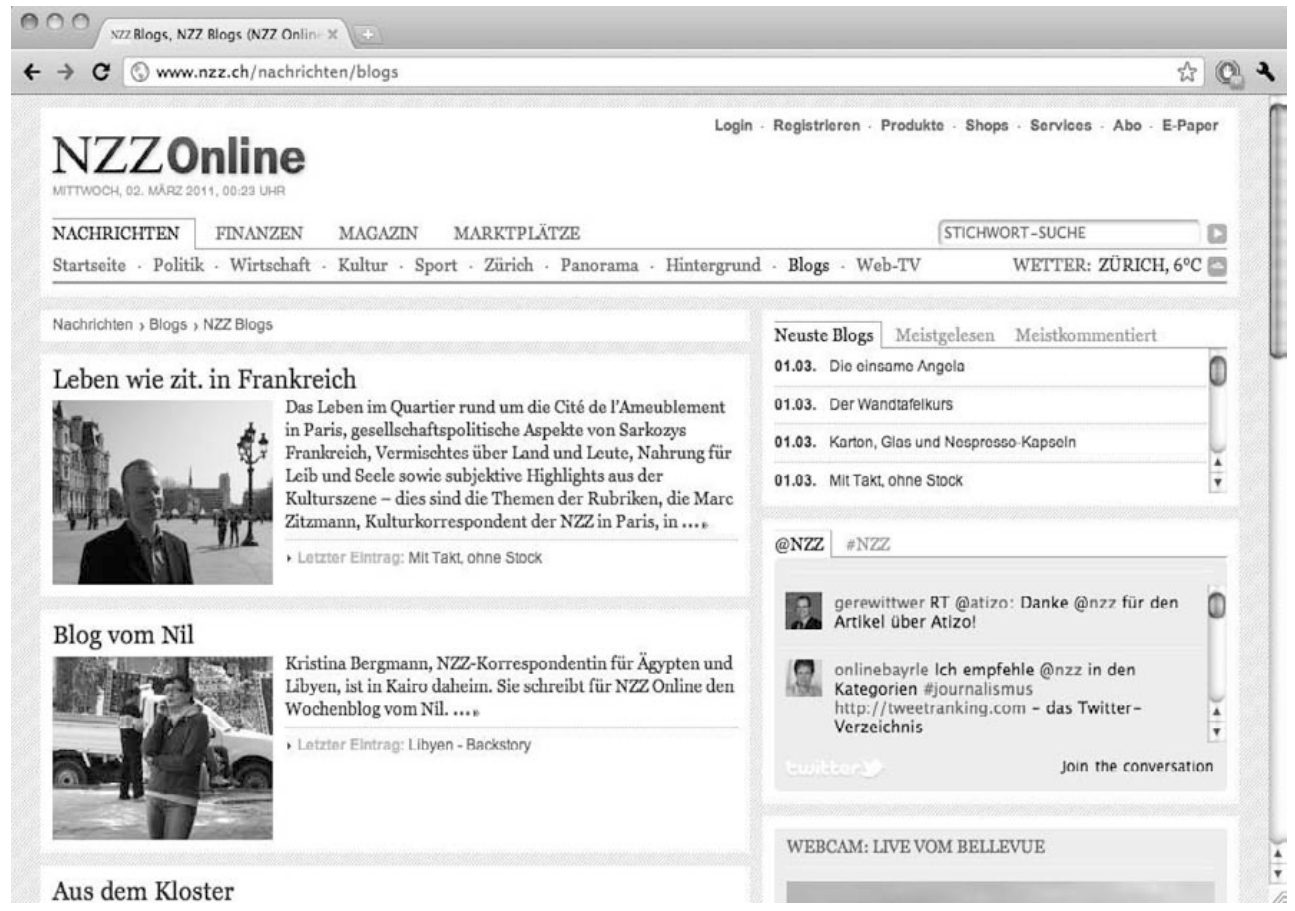

Abb. 3: Neue Zürcher Zeitung - Onlineausgabe, Rubrik Blogs

lokalen Pressediensten. Bürgerjournalismus ist ein Schlagwort, das schon seit einigen Jahren im Zusammenhang mit privaten Aktivitäten auf Blogs kursiert. Mit der Ausweitung und dem neuen Verständnis des Begriffs "Blog « und der eindrücklichen Entwicklung der Benutzerzahlen der neuen Onlineplattformen hat dieser Begriff eine Berechtigung erlangt.

\subsection{Blogger als Medienpartner}

In Abgrenzung der Anwender von sozialen Netzwerken und Bloggern, die ein Weblog per se als Gefäß für die Veröffentlichung von regelmäßigen Publikationen verwenden, zeigt sich eine veränderte Haltung der Tagespresse gegenüber privaten Autoren. Blogs sind inzwischen eine regelmäßig genutzte Informationsquelle für Journalisten: Politik, Technologie, Lifestyle sind beispielhafte Themen, die gerne in der Tagespresse aufgenommen und referenziert werden. Aber auch für kommerzielle
Kommunikation sind Blogs mit einer größeren Reichweite und Leserschaft mittlerweile interessant. Die Herausgeber werden auf Augenhöhe mit den Journalisten mit Informationen bedient. Über Presseverteiler von Unternehmen und PR-Agenturen werden Blogger immer häufiger zu Pressekonferenzen und Produkteeinführungen eingeladen oder mit Rezensionsexemplaren von Mobiltelefonen, Digitalkameras oder anderen Alltagsprodukten versorgt. Dies natürlich in der Hoffnung, dass die Blogger eine wohlgesinnte Kritik veröffentlichen würden.

Je schneller ein Medium, umso interessanter ist es für die Berichterstattung oder als Informationsquelle in Echtzeit. MicrobloggingDienste haben in vielen Fällen einen enormen Zeitvorteil bewiesen. Gemäß [Patalong 2009] wurde die Notlandung eines Flugzeugs auf dem Hudson River als Erstes von einem Augenzeugen mittels Handyfoto auf Twitter dokumentiert. Das Bild wurde im Anschluss von vielen 
Fernsehstationen verwendet, da keine Journalisten vor Ort waren.

Es folgen nun zusammengefasst ein paar Handlungsempfehlungen für die Praxis.

\section{Handlungsempfehlungen für die Praxis}

Die strikte Abgrenzung von traditionellen Weblogs und sozialen Medienplattformen verwässert im Web 2.o zusehends. Jedoch verdrängen soziale Netzwerke traditionelle Blogs nicht, sondern erweitern die Kommunikationsmöglichkeiten und Reichweite dieser sogar noch. Einzig die Anzahl aktiver Blogs und die Publikationsfrequenzen nehmen tendenziell eher $a b$.

Die Verschmelzung der sozialen Netzwerke mit den originären Weblogs schafft einen attraktiven Rahmen, um die persönliche Reputation im Internet auf- oder auszubauen. Zwei Faktoren spielen dabei eine wesentliche Rolle:

1. Dank der hohen Rangierung von Blogs in den Google-Trefferlisten eignen sich diese als primäre Plattform für einen eigenen Auftritt im Web wesentlich besser als vergleichsweise statische Webseiten oder reine ContentManagement-Systeme-idealerweise in Kombination mit einer eigenen Internetadresse (z.B. im Format: www.vornamenachname.ch).

2. Soziale Netzwerke begünstigen die weitere Verbreitung und somit die Reichweite der eigenen Blogbeiträge.

Eine gute Strategie ist dabei der Vorsatz, die ersten Google-Trefferseiten mit eigenen Inhalten zu bestücken. Dazu empfiehlt es sich, in jedem Fall mit dem vollen realen Namen und nicht etwa mit einer Abkürzung oder gar einem Pseudonym zu operieren. Darüber hinaus ist ein Konzept gefragt: Mit welchen Inhalten oder Themen soll die eigene Person im Web assoziiert werden? Bestimmte Fachthemen, Freizeitbeschäftigungen, ehrenamtliche Tätigkeiten oder gar Vereinsaktivitäten? Schlussendlich bildet im Web 2.0 nicht eine einzelne Webseite die
Reputation, sondern die Anzahl der selbst verfassten Blog-, Facebook- und Twitter-Beiträge in Kombination mit den dabei entstandenen Kommentaren und Dialogen - wohlgemerkt über einen längeren Zeitraum hinweg.

Bezeichnenderweise sind nicht die Benutzer selbst die Trendsetter, sondern die Plattformen und deren Konzepte per se. Sie prägen eine neue Kommunikationskultur, die neue Paradigmen geschaffen hat: ernsthafter Dialog statt Sendermentalität, das Teilen von Wissen und Inhalten (z.B. durch Veröffentlichung unter Creative-Commons-Lizenzen) anstelle von $\mathrm{Ab}$ grenzung durch Barrieren wie Passwörter oder nur das auszugsweise Zurverfügungstellen. Generell mag das schlussendlich das Erfolgsrezept für den Einsatz dieser neuen Medien sein, denn Technik und Inhalte waren schon zu Beginn der Web-Ära wichtig. Heute sind darüber hinaus auch kulturelle Aspekte in der Nutzung entscheidend: Authentizität, Präsenz, Relevanz und die Bereitschaft zum Austausch von Wissen und zu echtem Dialog mit der Online-Gemeinschaft.

\section{Literatur}

[Allemann 2010] Allemann, D.: Amnesty International: Social Media helfen Libyen-Geiseln, 2010, http://bernetblog.ch/2010/02/04/amnesty-international-social-media-helfen-libyen-geiseln/; Zugriff am 01.03.2011.

[Borgerding 2010] Borgerding, B.: Nestlé in Erklärungsnot, 2010, http://blog.greenpeace.de/blog/ 2010/03/18/nestle-in-erklaerungsnot/; Zugriff am 01.03.2011.

[Bourdieu 1983] Bourdieu, P.: Ökonomisches Kapital Kulturelles Kapital - Soziales Kapital. In: Kreckel, R. (Hrsg.): Soziale Ungleichheiten. Schwartz, Göttingen, 1983, S. 183-198.

[Hutter 2010] Hutter, T.: Facebook: Infografik und demographische Daten Deutschland, Österreich und Schweiz per Oktober 2010, www.thomashutter.com/index.php/2010/10/facebook-infografikund-demographische-daten-deutschland-osterreich-und-schweiz-per-oktober-2010/; Zugriff am 01.03.2011. 
[Ingenhoff \& Meier 2010] Ingenhoff, D.; Meier, A.: Webplattformen zur Community-Bildung. In: Heilmann, H. (Hrsg.): Humane Nutzung der Informationstechnologie. Integrata Stiftung, 2010 (in print).

[Patalong 2009] Patalong, F: Da ist ein Flugzeug im Hudson River. Verrückt. Spiegel Online, www.spiegel.de/netzwelt/web/o, 1518,601588,o0. html; Zugriff am 01.03.2011.

[Pfeiffer 2009] Pfeiffer, T.: Deutsche Twitterumfrage 2.0, 2009, http://twitterumfrage.de/dtu1.php; Zugriff am 01.03.2011.

[Portmann 2008] Portmann, E.: Informationsextraktion aus Weblogs - Grundlagen und Einsatzmöglichkeiten der gezielten Informationssuche. VDM, Saarbrücken, 2008.

[Schmidt \& Wilbers 2006] Schmidt, J.; Wilbers, M.: "Wie ich blogge?!" 2005. Berichte der Forschungsstelle "Neue Kommunikationsmedien«, 2006, Nr. 06-01, www.kowi.uni-bamberg.de/fonk/pdf/fonkberichto6o1.pdf; Zugriff am 01.03.2011.
[Sobel 2010] Sobel, J.: The State of the Blogosphere 2010, WHAT: Topics and Trends - Day 2 SOTB 2010, http://technorati.com/blogging/article/ what-topics-and-trends-day-2/; Zugriff am 01.03.2011.

Edy Portmann M.Sc. Universität Fribourg Departement für Informatik Boulevard de Pérolles 90 $\mathrm{CH}-1700$ Fribourg edy.portmann@unifr.ch http://diuf.unifr.ch/is

Eidg. dipl. Informatiker Ralph Hutter MBA Namics AG

Bederstr. 1

$\mathrm{CH}-8002$ Zürich

ralph.hutter@namics.com www.namics.com 